Vol. 5, No. 2, 2020

\title{
IMPROVEMENT OF ADSORPTION PROCESSES OF WASTEWATER TREATMENT FROM NICKEL IONS
}

\author{
Ihor Petrushka, Kateryna Petrushka, Bohdanna Bliatnyk \\ Lviv Polytechnic National University, \\ 12, S. Bandery Str., Lviv, 79013, Ukraine \\ petim@ukr.net
}

https://doi.org/10.23939/ep2020.02.083

Received: 06.04.2020

C Petrushka I., Petrushka K., Bliatnyk B., 2020

\begin{abstract}
The sorption properties of natural and complex sorbents for neutralization of $\mathrm{Ni}^{2+}$ ions from the water environment were studied. The isotherm of the adsorption of $\mathrm{Ni}^{2+}$ ions on complex sorbents (activated bentonite to shungite is 1:1) according to the Langmuir and Freundlich's models was drawn; the type of adsorption isotherms according to the S. Brunauer's classification was established. The values of the maximum sorption capacity of $\Gamma_{\max }$ sorbents relative to $\mathrm{Ni}^{2+}$ ions were calculated. Based on the calculated coefficients of the Langmuir and Freundlich's equations, the specificity of the process of sorption of Nickel ions with the formation of a monomolecular layer was determined. The dependences of the degree of absorption of $\mathrm{Ni}^{2+}$ ions by complex sorbents on the duration of the sorption process were obtained. The ratio of solid state of matter (comprehensive sorbent) to liquid was experimentally determined.
\end{abstract}

Key words: environmental security, natural sorbents, sorption isotherm, wastewater adsorption.

\section{Introduction}

Ukraine is one of the least water-supplied countries in Europe since the reserves of local river flow resources per person are about 1.0 thousand $\mathrm{m}^{3}$ per year. The main cause of surface water pollution is the discharge of untreated and insufficiently treated industrial wastewater.

More than a third of it is wastewater contaminated with heavy metals. These components have a negative impact on living organisms, as they have cumulative and toxic properties, and make it difficult to operate natural and wastewater treatment plants in localities. Sources of dangerous components are not only large and mediumsized industrial facilities but also natural dumps, in particular, used batteries containing mercury, cadmium, lead, tin, nickel, zinc, magnesium, and other chemical elements and compounds. In landfills, under the influence of atmospheric factors, batteries are quickly destroyed, and the substances in their composition evaporate and are washed away. Through water and air, toxic metals enter living organisms, where they cause damage, impair reproductive abilities, and cause genetic changes and cancer. Over time, the toxic substances that the battery releases accumulate in the human body. Chromium, Nickel, and Cadmium are accumulated in the kidneys, copper - in the gastrointestinal tract, mercury affects the central nervous system and the kidneys.

Removal of Nickel ions from liquid solutions and the study of the sorption mechanism are covered in numerous scientific publications of domestic and foreign scientists $[1-7,10]$

The most promising method of wastewater treatment is sorption technology, which is widely used in various industries. Currently, there is considerable experience in using natural clay minerals and their modified forms for wastewater treatment from heavy metal ions.

The prospect of using natural minerals in technological processes of wastewater treatment is due not only to their sufficiently high sorption capacity but also to the existence of effective methods of improving the adsorption properties of minerals and the nature of their surface by modification.

In addition to traditional natural sorbents (bentonite, palygorskite, glauconite), which are used for wastewater treatment from heavy metal ions, shungite is also interesting as a mineral by its sorption capacity.

Shungite is a rock that contains silicon dioxide. The mineral is widely used in various areas of human 
activity, in construction and metallurgy. But the physical and chemical properties of shungite are so unique that it began to be used in alternative medicine and for water purification. In nature, the mineral is formed as sediment of organic origin. Shungite is a transitional stage of graphite, which causes the dark color of the rock. Gray and brown minerals are less common $[8,9]$.

Shungite rocks are unique in composition, structure, and properties. They are an unusual natural composite by structure - a uniform distribution of highly dispersed crystalline silicate particles in an amorphous carbon matrix. The average size of silicate particles is about $1 \mu \mathrm{m}$. The average composition of the rock deposit is $30 \%$ of carbon and $70 \%$ of silicates. The rocks are characterized by high strength, density, chemical resistance, and electrical conductivity. They have some unusual physical, chemical, physical-chemical, and technological properties [8,9].

Chemical composition of shungite (mass \%) is: $\mathrm{Al}_{2} \mathrm{O}_{3}-4.05 \% ; \mathrm{Fe}_{2} \mathrm{O}_{3}-1.01 \% ; \mathrm{Fe}_{2} \mathrm{O}-0.32 \% ; \mathrm{K}_{2} \mathrm{O}-1.23 \%$; $\mathrm{CaO}-0.12 \%$; $\mathrm{SiO}_{2}-6.46 \%$; $\mathrm{MgO}-0.56 \%$; MnO-0.12\%; $\mathrm{Na}_{2} \mathrm{O}-0.36 \%$; $\mathrm{TiO}_{2}-0.24 \% ; \mathrm{P}_{2} \mathrm{O}_{3}-0.03 \% ; \mathrm{Ba}-0.32 \%$; $\mathrm{B}-0.004 \%$; V-0.015 \%; Co-0.00014\%; Cu-0.0037\%; Mo-0.0031\%; Ni-0.0085\%;Pb-0.0225\%;S-0.37\%; St-0.001\%; C-26.26 \%; Cr-0.0072 \%; Zn-0.0067\%; $\mathrm{H}_{2} \mathrm{O}-2.18 \%$.

The basic mass (up to $99 \%$ ) of shungite is represented by non-crystalline carbon, the defining feature of which is the globular structure. It is based on a ball - a hollow multilayer formation with dimensions up to $10 \mathrm{~nm}$. The marked curvature of graphite layers indicated in the article allowed the authors to make a conclusion about the fullerene-like structure of shungite carbon [8]. Mineral components are represented by finely dispersed (from 1 to $10 \mu \mathrm{m}$ ) crystals of mica, quartz, albite, etc. This structure allows us to consider shungite as a natural composite material and provides for the possibility of displaying its unique sorption properties [9].

An important task in studying the sorption properties of sorbents is the process of modeling and predicting the mechanism of pollutants absorption from liquid media [10-12].

Therefore, studies aimed at improving the adsorption processes of wastewater treatment from used batteries that contain mercury, cadmium, lead, tin, nickel, zinc, magnesium, etc. are relevant and essential for improving the environmental security of the water environment.

The aim of this work is to increase the level of environmental security of the hydrosphere by improving the adsorption processes of the liquid medium treatment from contamination with complex natural sorbents to purify wastewater from components of used batteries, in particular $\mathrm{Ni}^{2+}$ Nickel.

\section{Experimental part and results}

Adsorption processes are characterized by different forms of equilibrium Isotherms. Many adsorption systems are characterized by the shape of the Langmuir and Freundlich's isotherms. A characteristic feature of this type of adsorption isotherms is that at a certain value of the component concentration in a liquid medium, the velocities of mass transfer in the forward and reverse directions are equal, that is, the equilibrium concentration in the solid phase reaches a constant value.

Quantitatively, the degree of adsorption is determined by the excess of the substance at the interface of the phases compared to the equilibrium amount of this substance in the solution. By comparing the values of the initial concentration of cation in the solution with a residual concentration of metal ions after contact of the solution with the sorbent, it is possible to draw a conclusion about the adsorption property of the sorbent relative to ions and properties of the sorbent itself.

In order to obtain an optimal ratio between sorbents (activated bentonite to shungite), experimental studies of their sorption capacity in relation to $\left(\mathrm{Ni}^{2+}\right)$ were conducted.

To obtain the same granulometric composition of complex sorbents, they were first crushed in a laboratory ball mill with steel layers then, by sieve analysis, they were brought to a uniform granulometric composition with a grain size of up to $0.3 \mathrm{~mm}$.

Experimental determination of the adsorption size and the degree of extraction of the components from the solution was determined by the dependencies $(1,2)$ :

$$
\begin{aligned}
& \Gamma=\frac{\left(\mathrm{c}_{\mathrm{in}}-\mathrm{c}_{\mathrm{eq}}\right)}{\mathrm{m}_{\mathrm{sorb}}} \cdot \mathrm{W} \\
& \alpha=\frac{\left(\mathrm{c}_{\mathrm{in}}-\mathrm{c}_{\mathrm{eq}}\right)}{\mathrm{c}_{\mathrm{in}}} \cdot 100 \%,
\end{aligned}
$$

where $c_{i n}$ and $c_{e q}$ are the initial and equilibrium concentrations of the component in the solution $\left(\mathrm{mg} / \mathrm{dm}^{3}\right) ; \mathrm{m}_{\text {sorb }}$ is the mass of the sorbent $(\mathrm{g}) ; \mathrm{W}$ is the volume of the solution (1).

In practice, Freindlich's empirical equation is used for analysis and calculations:

$$
\Gamma=\beta \cdot \mathrm{C}^{1 / n}
$$

where $\beta$ and $1 / n$ are constants; $\mathrm{C}$ is the equilibrium concentration $\left(\mathrm{mg} / \mathrm{dm}^{3}\right)$.

The constants of the Freundlich's equation are found by the logarithm problem equation (3) and by constructing a graphical dependency:

$$
\mathrm{g} \Gamma=\ln \beta+\frac{1}{\mathrm{n}} \lg \mathrm{C} .
$$

To determine the maximum adsorption $\left(\Gamma_{\max }\right)$, the dependence $1 / \mathrm{G}=\mathrm{f}(1 / \mathrm{C})$ is constructed, which makes it possible to determine the $\Gamma_{\max }$ that corresponds to the complete saturation of the surface layer of the adsorbent. 
Based on the prepared model solutions, $\mathrm{Ni}^{2+}$ adsorption isotherms were obtained on natural and complex sorbents (Fig. 1).

The graphic data show that the highest sorption capacity in relation to $\mathrm{Ni}^{2+1}$ in static conditions has a complex sorbent - activated bentonite to shungite" in the ratio 1:1.

To determine the parameters of intensity of the adsorption processes and establish the type of adsorption isotherms, the experimental data were interpreted in the framework of the Langmuir and Freundlich's theoretical models.

The obtained isotherms of $\mathrm{Ni}^{2+}$ adsorption on complex sorbents (Fig. 2) belong to the first type according to the S. Brunauer's classification with a characteristic bend relative to the concentration axis at the initial stage of sorption followed by the formation of a plateau, which characterizes the end of the saturation period of the sorbent surfaces.

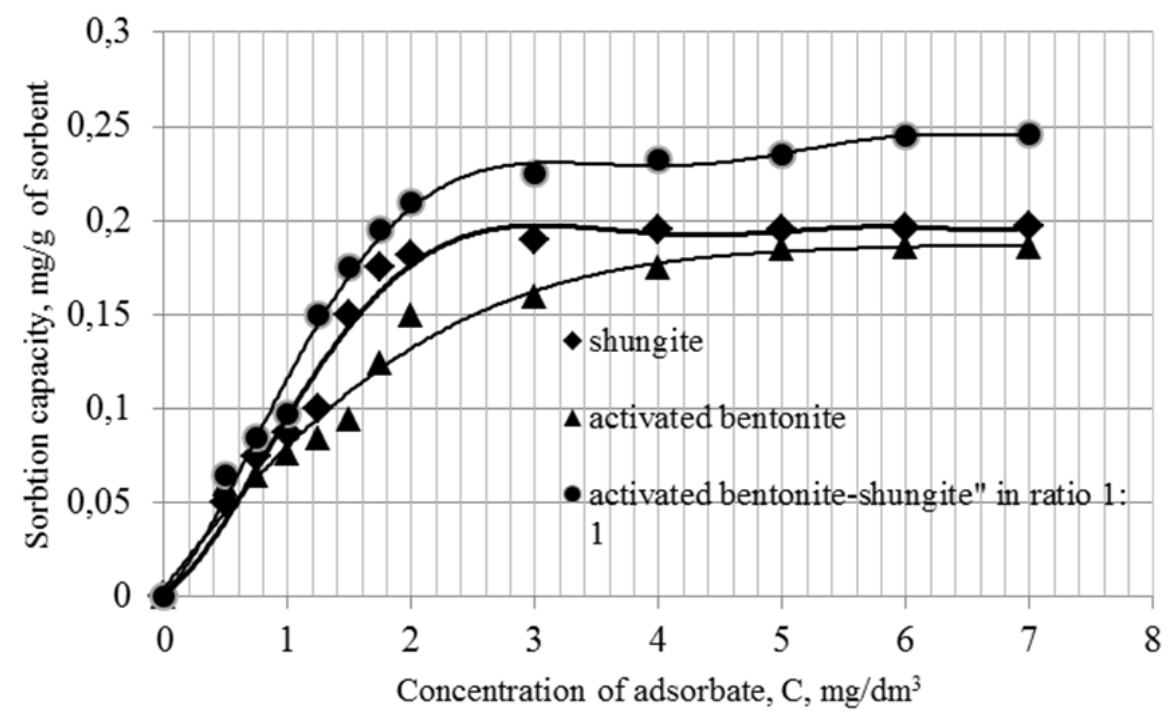

Fig. 1. Isotherms of $\mathrm{Ni}^{2}+$ adsorption by natural sorbents

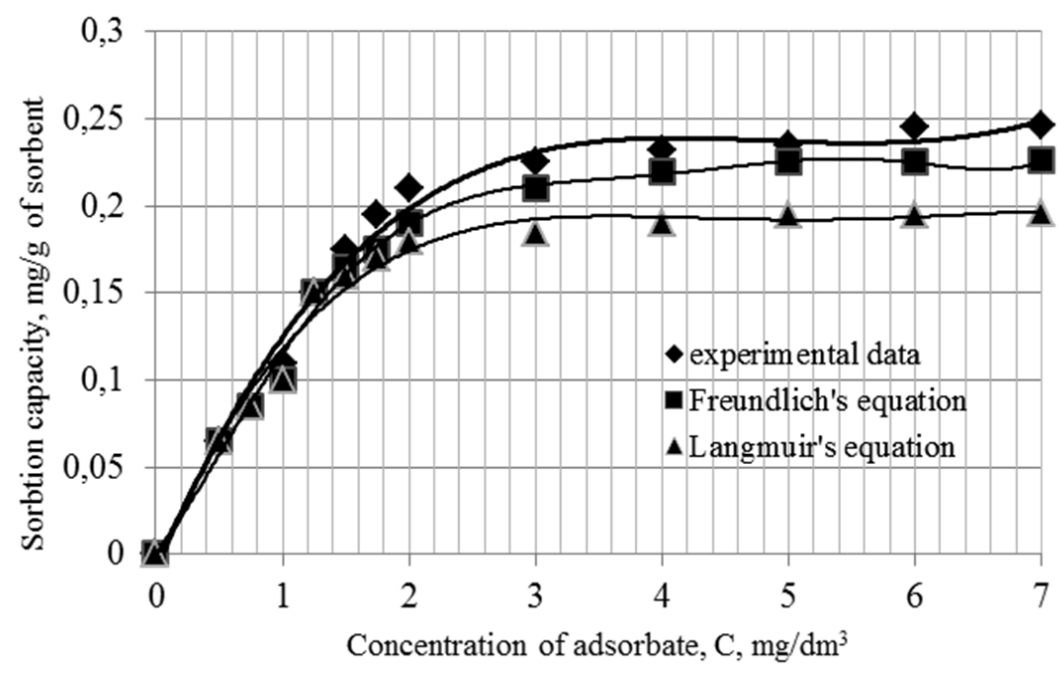

Fig. 2. Isotherm of nickel sorption by a complex sorbent (activated bentonite:shungite) according to the results of the experiment and the ratio of the Freundlich and Langmuir's equations

Based on the comparison of the approximation value $\left(\mathrm{R}^{2}\right)$ (Table 1) it has been established that the experimental data obtained are described with sufficient accuracy by the Freundlich's equation, and with less accuracy they are described by the Langmuir equation, which in both cases indicates that sorption occurs with the formation of a monomolecular layer. High values of the determination ratio indicate significant chemical heterogeneity of the surface of complex sorbents, which causes uneven distribution of active centers on the surface of materials and affects the degree of filling of the adsorption layer (Table 1). 


\section{Parameters of $\mathrm{Ni}^{2+}$ complex sorbent adsorption isotherms}

\begin{tabular}{|c|c|c|c|c|c|c|}
\hline \multirow{2}{*}{ Adsorptive } & \multicolumn{3}{|c|}{ Langmuir's equation } & \multicolumn{3}{c|}{ Freindlich's Equation } \\
\cline { 2 - 6 } & $\begin{array}{c}A_{\max }, \\
\mathrm{mg} / \mathrm{g}\end{array}$ & $C$ & $\mathrm{R}^{2}$ & $\begin{array}{c}m, \\
\mathrm{mg} / \mathrm{g}\end{array}$ & $1 / n$ & $\mathrm{R}^{2}$ \\
\hline \multicolumn{6}{|c|}{ Activated bentonite-shungite $(1: 1)$} \\
\hline $\mathrm{Ni}^{2+}$ & 0.2492 & 1.5425 & 0.9277 & 0.12689 & 0.385 & 0.9658 \\
\hline
\end{tabular}

The ratio of the Langmuir and Freundlich's equations is calculated to give an idea of the nature of the process of sorption of $\mathrm{Ni}^{2+}$ ions by the value of the maximum sorption capacity and the affinity of the sorbents.

As part of the study of the adsorption properties of complex sorbents based on activated bentonite and shungite relative to nickel ions, it was found that to achieve the maximum degree of treatment at the minimum cost of the sorption material, the necessary ratio of solid to liquid is $1: 30$. The mass of the sorbent was 10,20 and $30 \mathrm{~g} / \mathrm{dm}^{3}$. The dynamics of changes in the concentration of $\mathrm{Ni}^{2+}$ ions $\left(\mathrm{S}_{\mathrm{in}}=2.0 \mathrm{mg} / \mathrm{dm}^{3}\right)$ depending on the time of interaction of the model solution with a natural sorbent are shown in Fig. 3.

The obtained results indicate that the optimal sorption conditions are observed at a ratio of solid to liquid as 1:30. It was found that when the concentration of the sorbent increases by several times, the degree of extraction increases only by $2-9 \%$ and does not exceed $90.5 \%$ of the initial concentration.

The experiments have shown that the concentration of the sorbent more than $30 \mathrm{~g} / \mathrm{dm}^{3}$ does not lead to a significant decrease in the concentration of $\mathrm{Ni}^{2+}$ ions. It was found that the optimal amount of clay material of $30 \mathrm{~g} / \mathrm{dm}^{3}$ in $5 \mathrm{~min}$. reduces the nickel concentration from $2 \mathrm{mg} / \mathrm{dm}^{3}$ to $0.27 \pm 0.04 \mathrm{mg} / \mathrm{dm}^{3}$, usually for 30 minutes. The sorption stabilization process happens, after which a significant decrease in the concentration of $\mathrm{Ni}^{2+}$ ions does not occur. The maximum degree of treatment is $87 \%$, which confirms the effectiveness of using a complex sorbent for the treatment of wastewater contaminated with $\mathrm{Ni}^{2+}$ ions.

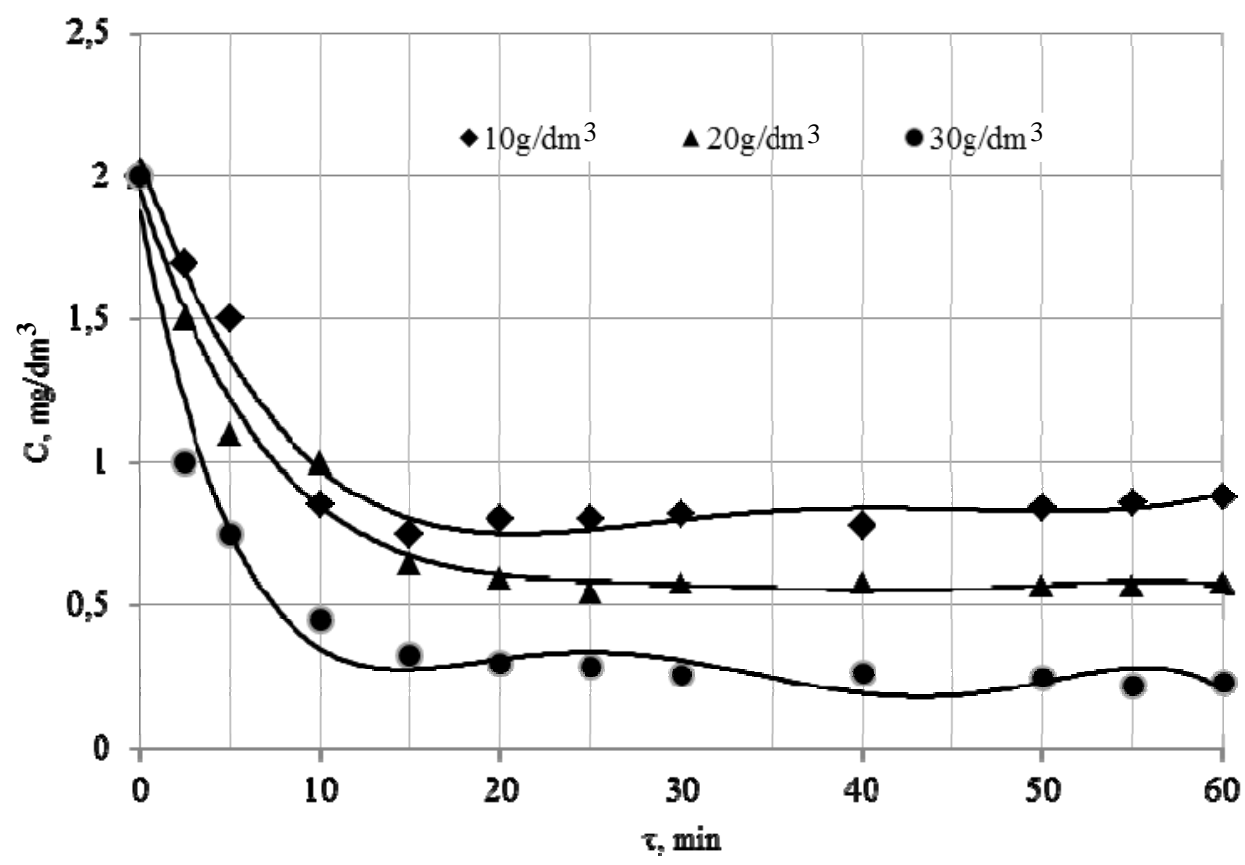

Fig. 3. Changes in the concentration of $\mathrm{Ni}^{2+}$ ions in the solution at different concentrations of the complex sorbent: $1-10 \mathrm{~g} / \mathrm{dm}^{3} ; 2-20 \mathrm{~g} / \mathrm{dm}^{3} ; 3-30 \mathrm{~g} / \mathrm{dm}^{3}$

\section{Conclusions}

The isotherm of the adsorption of $\mathrm{Ni}^{2+}$ ions on complex sorbents (activated bentonite to shungite (1:1) according to the Langmuir and Freundlich's models was obtained; the type of adsorption of isotherms according to the S. Brunauer's classification was established. The value of the maximum sorption capacity of $\Gamma_{\max }$ sorbents relative to $\mathrm{Ni}^{2+}$ ions, which is $0.24 \mathrm{mg} / \mathrm{g}$, has been established. The calculated coefficients of the Langmuir and Freundlich's equations made it possible to determine the nature of the process of sorption of $\mathrm{Ni}^{2+}$ ions with the formation of a monomolecular layer.

It was found that at the sorption of ions and $\mathrm{Ni}^{2+}$ on a complex sorbent, the most appropriate ratio of solid to liquid is 1:30, while the maximum decrease in 
concentration is observed after 5-10 minutes of the treatment process and is almost $94 \%$. After 30 minutes the sorption process stabilizes, after which a decrease in the concentration of $\mathrm{Ni}^{2+}$ ions is not significant.

\section{References}

[1] Malovanyy M., Vronska N., Koval I., Sakalova G.: Bull. Lviv Polytechnic National Univ.: Chem., technol. of substan. and their appl., 2013, 761, 280 (in Ukrainian).

[2] Kontsur A., Sysa L., Shevchuk L.: Physics and chemistry of solid state, 2018, 19 (2), 191. https://doi.org/10.15330/pcss.19.2.191-196

[3] Riabchevskyi O., Matvieieva O.: Ecolog. Safety and Balanc. Use of Res., 2015, 2, 106.
[4] Gaouar- Yadi M., Tizaoui K., Gaouar-Benyelles N., Benguella B.: Indian J. Chem. Technol., 2016, 23, 204.

[5] Sabino De Gisi, Giusy Lofrano, Grassi M., Notarnicola M.: Sustainable Mater.Technol., 2016, 9, 10.

[6] Zhao X., Song L., Zhang Z., Wang R. J. Fu: J. Molec. Struct., 2011, 986, 68.

[7] Repo E., Petrus R., Sillanpaa M., Warchoł J. K.: Chem. Eng. J., 2011, 172(1), 376.

[8] Shpylevskyi M., Shpylevskyi Ye., Stelmakh V.: J. Eng. Physics, 2001, 76 (6), 25.

[9] Berezkin V.: Uhlerod. Zamknuti nanochastyny, makrostruktury, materialy. "AtrErho", 2013.

[10] Sydorchuk O., Humnytskyi Ya.: Eastern-European J. Enterprise Technol., 2013, 4/10 (64), 19.

[11] Petrushka I., Malovanyy M., Petrushka K.: Visn. KrNU im. M. Ostrohradskoho 2013, 2, 129.

[12] Petrushka I., Ruda M., Hyvliud A., Koval N.: Sci. Bull. UNFU, 2019, 29 (3), 64. https://doi.org/10.15421/ 40290314 